\title{
The adaptive architecture is shaped by population ancestry and not by selection regime
}

\author{
Kathrin A. Otte ${ }^{1, \#}$, Viola Nolte ${ }^{1, \#}$, François Mallard ${ }^{1,2}$, Christian Schlötterer ${ }^{1, *}$ \\ ${ }^{1}$ Institut für Populationsgenetik, Vetmeduni Vienna, Vienna, Austria \\ ${ }^{2}$ Present address: Institut de Biologie de l'École Normale Supérieure, CNRS UMR 8197, \\ Inserm U1024, PSL Research University, F-75005 Paris \\ "both authors contributed equally \\ *corresponding author: christian.schloetterer@vetmeduni.ac.at
}

Key words: Experimental Evolution, Temperature Adaptation, Adaptive Architecture 
1 Abstract

2 Understanding the genetic architecture of adaptive phenotypes is a key question in

3 evolutionary biology. One particularly promising approach is Evolve and Resequence

4 (E\&R), which combines advantages of experimental evolution such as time series, replicate

5 populations and controlled environmental conditions, with whole genome sequencing.

6 The recent analysis of replicate populations from two different Drosophila simulans

7 founder populations, which were adapting to the same novel hot environment, uncovered

8 very different architectures - either many selection targets with large heterogeneity among

9 replicates or fewer selection targets with a consistent response among replicates.

10 Here, we exposed the founder population from Portugal to a cold temperature regime.

11 Although almost no selection targets were shared between the hot and cold selection

12 regime, the adaptive architecture was similar: we identified a moderate number of loci

13 under strong selection (19 selected alleles, mean selection coefficient $=0.072)$ and very

14 parallel responses in the cold evolved replicates. This similarity across different

15 environments indicates that the adaptive architecture depends more on the ancestry of the

16 founder population than the specific selection regime. These observations have a

17 pronounced impact on our understanding of adaptation in natural populations. 


\section{Introduction}

19 Adaptation of natural populations to environmental change may either occur from standing genetic variation or by the acquisition of new mutations. The relative importance of these two paths crucially depends on the underlying adaptive architecture (Barghi et al. 2020) of the focal trait. The adaptive architecture differs from the genetic architecture, which is inferred by QTL mapping and GWAS, by accounting for pleiotropic constraints as well as for the large body of deleterious mutations (Gazal et al. 2017; Zeng et al. 2018). Depending on the context, two different aspects of the adaptive architecture are emphasized. The focus is either the identity of specific loci/SNPs or the general characteristics of the adaptive architecture characterized by the number of contributing loci and their effect sizes and frequency in the focal population (Barghi et al. 2020).

29 Contributing loci are either identified by QTL/GWAS studies (Flint and Mott 2001; McCarthy et al. 2008) or with genomic selection scans, which apply statistical tests to detect selection

31 signatures from population polymorphism data (Storz 2005; Vitti et al. 2013). Some selection

32 scans assume that selection targets are shared among populations exposed to the same

33 environment, because such parallel signatures provide additional statistical support (Turner et al.

34 2010; Lee and Coop 2017; Harris and DeGiorgio 2020). Many selection targets were

35 successfully identified with these strategies and have contributed to our understanding of the

36 molecular basis of adaptation processes (e.g. Turner et al. 2010; Jones et al. 2012; Roesti et al.

37 2014; Reid et al. 2016; Johnson and Voight 2018). It is, nevertheless, not apparent to what extent

38 these results can be generalized, because most adaptive traits have a polygenic basis (Barton and

39 Keightley 2002; Sella and Barton 2019) and either only small allele frequency changes (Sella

40 and Barton 2019) or non-parallel responses are expected (Barghi et al. 2020).

41 The key concept of polygenic adaptation is that multiple loci are contributing to the phenotype,

42 and rather than focusing on particular loci, the collective effect of all loci needs to be considered

43 to estimate the phenotypic value of a given trait. This has important implications for the

44 understanding of the adaptive architecture (Barghi et al. 2020).

45 The infinitesimal model (Fisher 1918; Bulmer 1971; Barton et al. 2017) is the most extreme case 46 of polygenic adaptation and is frequently approximated by very many contributing loci, each of 
47 very small effect. When many loci are contributing to a phenotype under stabilizing selection,

48 any selection regime changing the trait optimum will result only in very small allele frequency

49 shifts (Bulmer 1971; Sella and Barton 2019) - almost impossible to detect with classic

50 population genetic tests (Pritchard et al. 2010; Field et al. 2016; Jain and Stephan 2017a).

51 Even when these conditions are relaxed and a distribution of effect sizes with some large effect

52 alleles is considered, no pronounced allele frequency changes are expected when the populations

53 are large and in mutation selection equilibrium: alleles with large effects are segregating at low

54 frequencies only and do not contribute much to the phenotypic variance of the population upon

55 which selection is operating (de Vladar and Barton 2014; Jain and Stephan 2017b). Theory

56 predicts that as the pool of contributing loci to the selected phenotype becomes smaller (i.e. a

57 decreased mutational target), larger allele frequency changes are expected that will progressively

58 be detected in population genetic analyses (Höllinger et al. 2019). Therefore, traits with an

59 intermediate number of contributing loci are particularly interesting, because the response of

60 these loci can be sufficiently strong to be detected in experiments while, at the same time, being

61 informative about polygenic adaptation: more loci are segregating in the population than

62 required to reach a new trait optimum (i.e. genetic redundancy).

63 The consequence of this genetic redundancy is that the contribution to the phenotype can be

64 highly heterogeneous for individual loci in differentiated populations if they vary in frequency.

65 This expectation nicely conforms with empirical data, mostly from QTL studies, which find

66 heterogeneous sets of contributing loci among different populations (Adeyemo et al. 2009; Wu et

67 al. 2013; Al Olama et al. 2014; Li and Keating 2014; Conte et al. 2015; Horikoshi et al. 2018;

68 Takata et al. 2019; Wojcik et al. 2019; Zan and Carlborg 2019; Hodonsky et al. 2020). In the

69 case of adaptation to a new trait optimum, genetically differentiated populations will adapt by

70 frequency changes of different sets of loci. Hence, for polygenic adaptation the identity of

71 individual selected loci is not very important to describe the adaptive architecture, rather

72 information about the number of loci, effect sizes and frequencies are needed to understand the

73 selective response.

74 Selection signatures not shared among natural populations are difficult to interpret, as the

75 distinction between population-specific selection targets and false positive/negative signals can 
76 be challenging given the high impact of a largely obscure demography on selection signatures

77 (Jensen et al. 2005; Stajich and Hahn 2005; Li et al. 2012; Lohmueller 2014; Pavlidis and

78 Alachiotis 2017; Johri et al. 2020). Experimental evolution, in contrast, provides the advantage

79 of replicate populations, which evolve from the same founder population under controlled

80 experimental conditions (Kawecki et al. 2012). The potential of experimental evolution to study

81 the genomic signatures of polygenic adaptation has, however, not yet been fully exploited since

82 most studies apply truncating selection. Thus, the contributing alleles experience continued

83 selection pressure throughout the entire experiment, causing a parallel selection response in the

84 replicate populations towards an extreme phenotype. Laboratory natural selection is a specific

85 experimental evolution design, where the evolving populations are exposed to a new

86 environment (Garland and Rose 2009). In contrast to truncating selection, populations are

87 expected to reach a new phenotypic optimum. In combination with whole genome sequencing, it

88 provides an interesting approach to study the adaptive architecture experimentally.

89 Two previous experimental evolution studies conducted in the same novel hot laboratory

90 environment revealed very different adaptive architectures (Mallard et al. 2018; Barghi et al.

91 2019). In the Portugal experiment, five strongly selected genomic regions were identified and

92 this selection signal was highly parallel across replicates. For the Florida experiment, 99

93 selection targets were identified and considerable heterogeneity was observed between the

94 replicates. One possible explanation for this different adaptive architecture is that the ancestral

95 trait optima differed between the two founder populations (Barghi and Schlötterer 2020), leading

96 to a more intense selection in the Portugal experiment, because it was less well adapted than the

97 Florida founder population to high temperatures. Alternatively, more large effect alleles may

98 have been segregating at higher frequencies in the Portugal founder population.

99 Here, we exposed replicate populations of the Portugal founders to a cold temperature regime to

100 shed more light on the different selection responses. Interestingly, we found very little overlap

101 between the genomic position of the selection targets in the hot and cold temperature regimes.

102 Most large effect loci detected in the hot environment did not respond in the cold, suggesting that

103 hot and cold temperature adaptation may be different traits, rather than a simple shift in optimum

104 of the trait 'temperature adaptation'. Nevertheless, adaptation to both, hot and cold, temperature 
105 regimes had a very similar adaptive architecture - with a comparable number of selection targets

106 and effect sizes. We conclude that the adaptive architecture differs between populations and may

107 be trait independent. We discuss to what extent this phenomenon can be explained by the

108 infinitesimal model.

\section{Results}

110 We studied the genetic architecture of cold adaptation in Drosophila simulans by combining 111 experimental evolution with whole genome re-sequencing (Evolve and Resequence, E\&R). Five

112 replicate populations originating from the Portuguese founder population described by Mallard et 113 al. (2018) evolved for more than 50 generations (about four years) in a cold temperature regime

114 with daily fluctuations between $10^{\circ} \mathrm{C}$ and $20^{\circ} \mathrm{C}$. Genome-wide allele frequencies were

115 determined in 10 generation intervals by sequencing pools of individuals (Pool-Seq (Schlötterer

116 et al. 2014)). Contrasting generation 0 with 51 we identified 6,527 SNPs, which changed in

117 frequency more than expected by genetic drift either across all five replicates (adapted CMH test

118 (Spitzer et al. 2020), 6,510 SNPs) or at least in one replicate (adapted $\chi^{2}$ test (Spitzer et al. 2020),

119 additional 17 SNPs). The X chromosome harbored only 142 SNPs. Such a low number of

120 candidate SNPs on this chromosome was not seen in other Drosophila E\&R studies that

121 observed similar numbers of candidate SNPs on the X chromosome and autosomes (Jha et al.

122 2015; Jha et al. 2016; Barghi et al. 2019; Kelly and Hughes 2019; Michalak et al. 2019).

123 The pronounced peak structure in the Manhattan plot (Figure 1A) indicates that many candidate

124 SNPs are not independent due to linkage (Nuzhdin and Turner 2013; Franssen et al. 2017a). We

125 accounted for this and employed a correlation-based haplotype reconstruction approach to

126 identify independently selected haplotype blocks based on their distinct trajectories (Franssen et

127 al. 2017a; Otte and Schlötterer 2017) and treated each of these haplotype blocks as a single target

128 of selection (Barghi et al. 2019). 
bioRxiv preprint doi: https://doi.org/10.1101/2020.06.25.170878; this version posted July 8, 2020. The copyright holder for this preprint (which was not certified by peer review) is the author/funder, who has granted bioRxiv a license to display the preprint in perpetuity. It is made available under aCC-BY 4.0 International license.

A

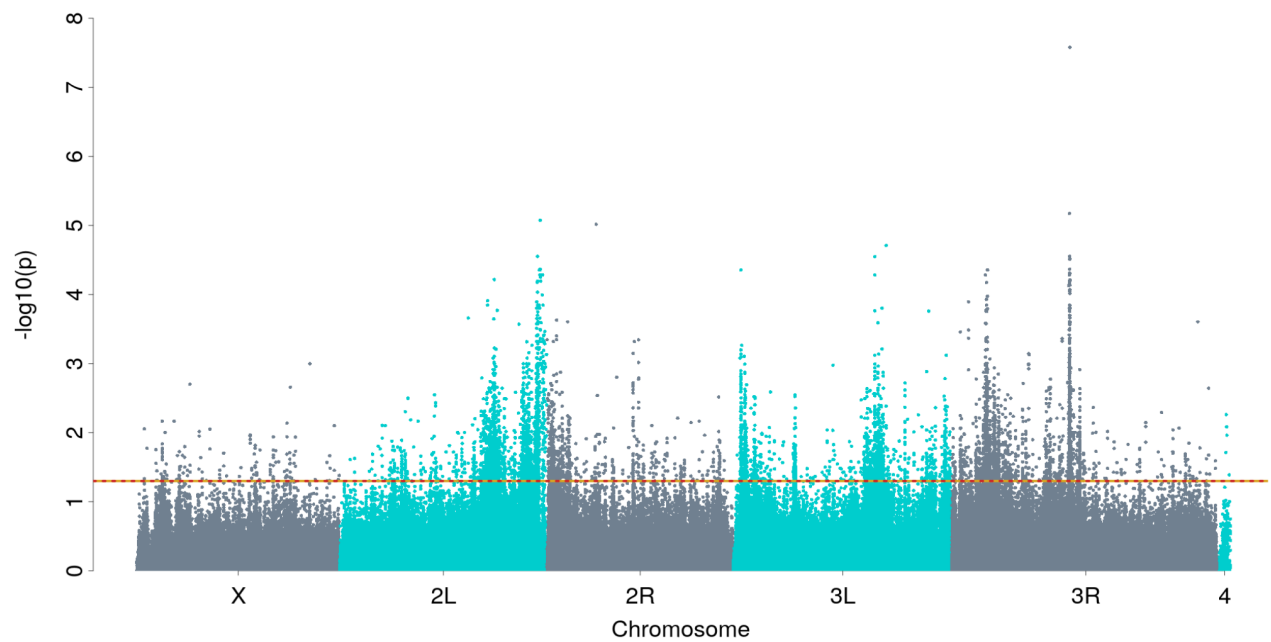

B

Chromosome 2

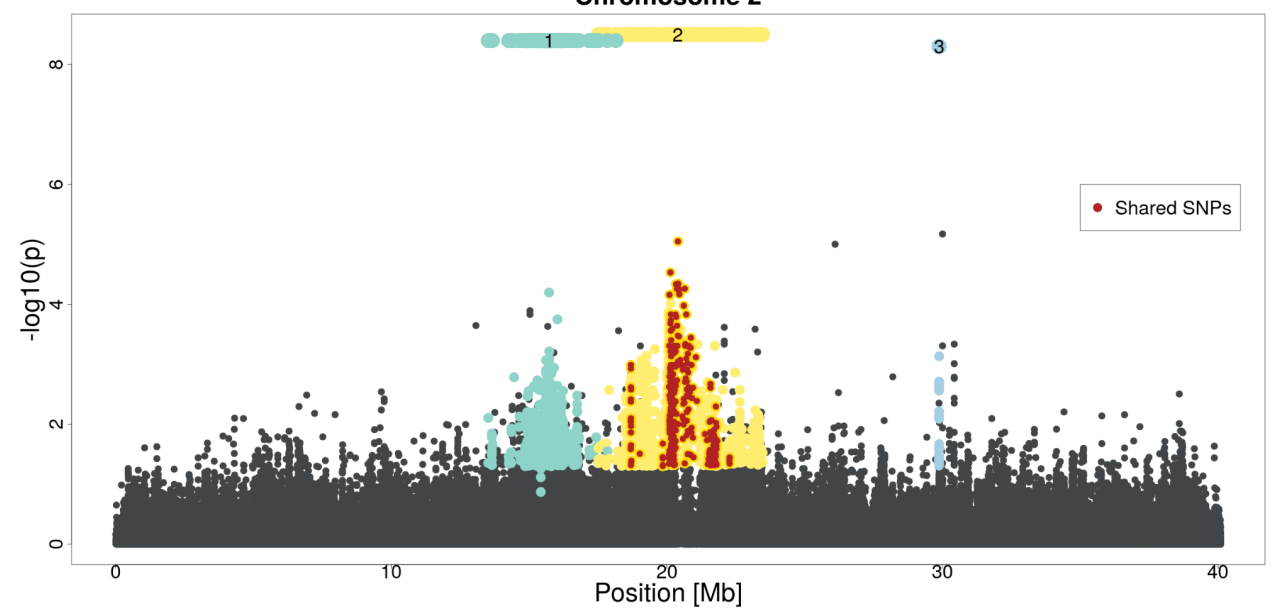

C

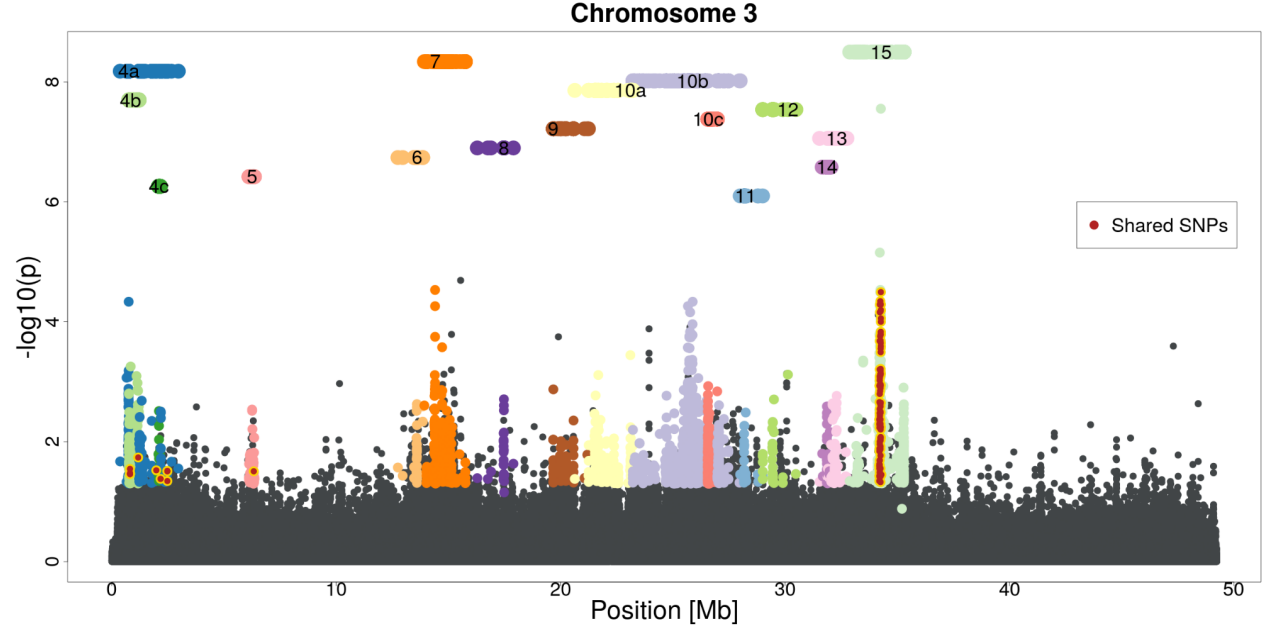


Figure 1: Manhattan plots of the genomic selection signature in response to cold temperature. A) p-values were obtained from an adapted CMH test (Spitzer et al. 2020) comparing the founder generation (F0) to the most advanced (F51) generation. The dotted line indicates the significance threshold (p-value $<0.05$ after correction for multiple testing). B, C) A close up of the Manhattan plot in panel A for chromosomes 2 and 3. Each selected haplotype block and the corresponding candidate SNPs are shown in a distinct color. Numbered bars above the Manhattan plot indicate the position of the selected haplotype blocks. Some blocks, which can be further subdivided by the analysis of earlier time points, are labeled with letters (e.g. 10a, 10b etc.). SNPs with significant allele frequency changes in both, hot and cold, selection regimes (shared SNPs) are colored in dark red.

\section{Selected haplotype blocks in the cold-evolved D. simulans population}

Because the small number of candidate SNPs precluded haplotype block reconstruction on the $\mathrm{X}$ chromosome, all 15 haplotype blocks were identified on the major autosomes (Figure 1B, 1C). The size of the haplotype blocks ranged from $5 \mathrm{~kb}$ to $6 \mathrm{Mb}$. Three of the 15 blocks were located on chromosome 2 while all others fell on chromosome 3.

The 15 selected haplotype blocks were identified by clustering SNPs with similar allele frequency trajectories in the five replicates and six time points. A conservative assumption is that each of the selected haplotype blocks contains one selection target. Nevertheless, multiple selection targets can recombine during the experiment onto a single haplotype block, which outcompetes the individual parental haplotype blocks (Otte and Schlötterer 2019). Such multiple-target haplotypes will dominate the later phases of the experiment and are considered as a single selected haplotype. To identify such cases, we repeated the haplotype block reconstruction with fewer generations - four time points up to generation 31 - and found that two reconstructed haplotype blocks could be further broken up: block 4 and 10 were split into three different blocks each (4a, 4b, 4c and 10a, 10b, 10c, see Figure 1C). This observation confirms that the number of inferred independent haplotype blocks is a conservative estimate of the number of selection targets, and we used the sub-blocks for subsequent analyses whenever applicable, analyzing a total of 19 selected alleles.

Selected haplotype blocks are characterized by a set of marker SNPs which show correlated allele frequency trajectories across replicates. Nevertheless, the correlation is not very stringent to account for sequence diversity among the haplotypes carrying the selection target (Otte and 
159 Schlötterer 2019). Hence, not all of the haplotype block marker SNPs describe the frequency

160 trajectory of the (unidentified) selection target equally well. Reasoning that SNPs with the most

161 pronounced allele frequency change are the best representatives of the selection target, we used

162 the 10\% most significant marker SNPs of each haplotype block and refer to them as the selected

163 allele. The frequency of each selected allele at every time point is determined as the median

164 frequency of these $10 \%$ most significant SNPs. We used only replicates with a selection

165 coefficient large enough to be significantly different from neutrality ( $p$-value $<0.05)$, therefore

166 we excluded replicates in which a given selected allele did not increase in frequency. The

167 starting frequencies were highly variable among the 19 selected alleles. We detected selected

168 alleles with starting frequency as low as 0.06 , but also as high as 0.4 (Figure 2A). The selection

169 coefficients were rather high and ranged from 0.04 to 0.11 (Figure 2A).

170 Overall, we noticed a striking relationship between starting frequency and selection coefficients.

171 Selected alleles starting with lower frequencies had higher selection coefficients than selected

172 alleles with higher starting frequencies. This relationship was significant when analyzing the full

173 set of 19 blocks including the broken-up haplotype blocks (i.e. replacing block 4 and 10 by sub-

174 blocks 4a, 4b, 4c and 10a, 10b, 10c) and was not significantly influenced by block size (linear

175 regression; factor: starting allele frequency $p=0.004$ and factor: block size $p=0.130$ ). The

176 inverse relationship between starting allele frequency and selection coefficient was robust with

177 respect to the definition of a selected allele (see Supplementary Figure S1).

178 While a negative correlation between frequency and effect size is expected by theory and has

179 been previously reported for GWAS (Eyre-Walker 2010; Simons et al. 2014; Mancuso et al.

180 2016; Zeng et al. 2018) and E\&R (Barghi et al. 2019) studies, it is important to note that a

181 Beavis-like effect (Beavis 1998; Xu 2003) may also contribute to this observation: alleles with

182 low starting frequencies require stronger selection to result in detectable allele frequency

183 changes than alleles starting from intermediate frequencies. 

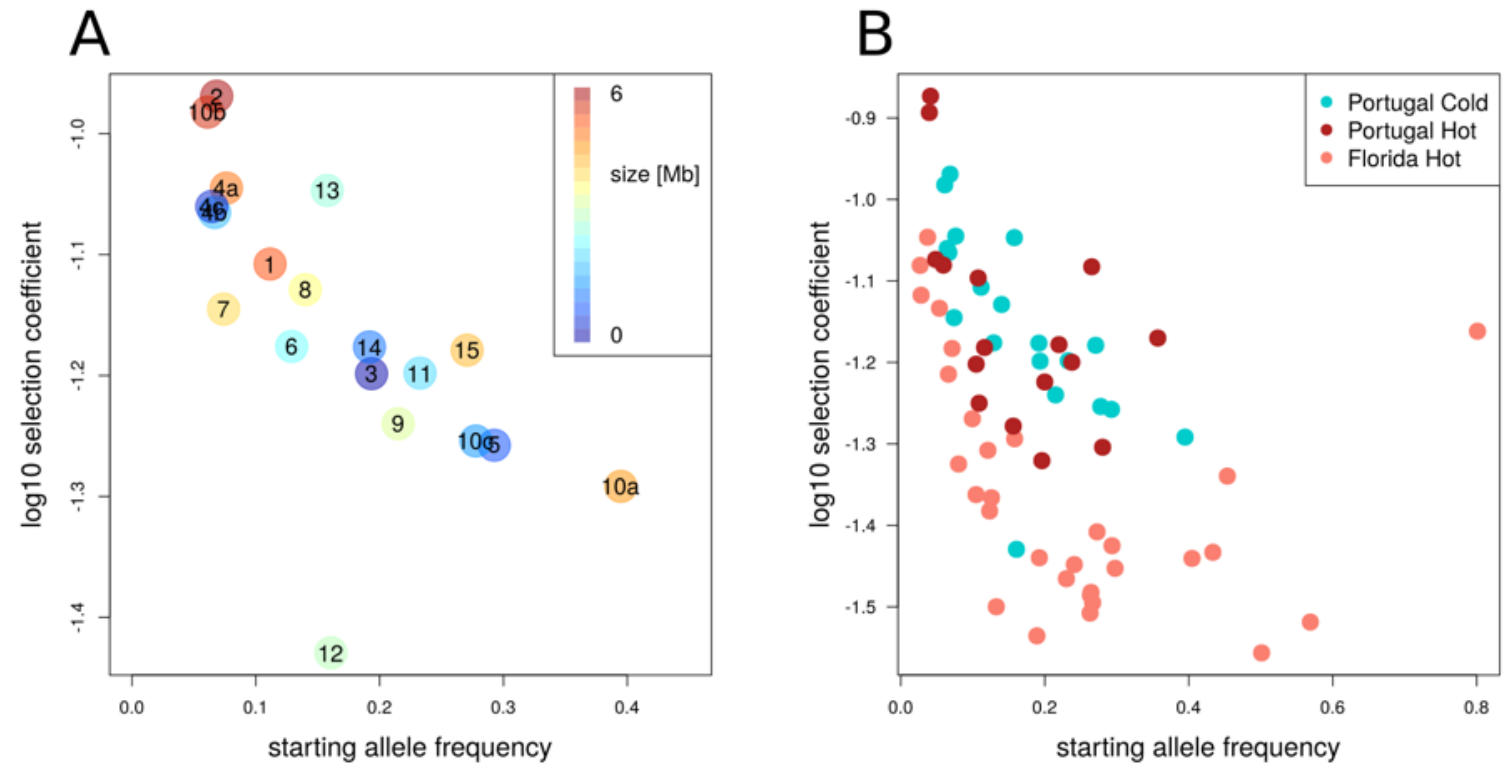

184 Figure 2: Inverse relationship between starting allele frequency and selection strength. For all

185 experiments, the selected alleles were identified using the same protocol and are based on the top $10 \%$

186 SNPs in reconstructed haplotypes. A) The relationship is shown for the Portugal cold experiment. The

187 color code reflects the size of the selected haplotype block, and starting allele frequencies and selection

188 coefficient of the selection targets are plotted on $\mathrm{x}$ - and $\mathrm{y}$-axis. The numbers relate to the selected

189 haplotype blocks from Figure $1 \mathrm{~B}$ and $1 \mathrm{C}$ with numbers indicating blocks detected in the analysis using all

190 time points. Sub-blocks resulting from an analysis using earlier time points are labeled with the letters a, b

191 or c. B) A qualitatively similar relationship between starting allele frequency of each haplotype block and

192 selection strength is found in the cold-evolved Portugal (blue), hot-evolved Portugal (red) and hot-

193 evolved Florida (pink) population. Nevertheless, the distribution in the Florida experiment was shifted

194 towards lower selection coefficients while the two temperature regimes in the Portugal population were

195 highly similar. We conclude that the adaptive architecture is population-specific but does not depend on

196 the temperature regime.

197 With a median size of $1.5 \mathrm{Mb}$, the selected haplotype blocks were rather large. The median

198 number of genes per selected haplotype block is 20 but it can reach up to 154 (see

199 Supplementary Figure S2) in the largest reconstructed block (block 2, see Figure 1B). The

200 smallest block contained only a single candidate gene (block number 3, $4.8 \mathrm{~kb}$ ). All 23 marker

201 SNPs were located within one intronic region of the gene M-spondin (mspo, FBgn0020269), an

202 extra-cellular matrix protein of Drosophila, putatively involved in muscle development (Bataille 
203 et al. 2010). The role of this gene in temperature adaption is not apparent and further studies are

204 required for a better understanding of this selection signature.

\section{Temperature-specific adaptation}

206 Replicates from the same Portuguese founder population were also exposed to a hot selection

207 regime fluctuating between $18^{\circ} \mathrm{C}$ and $28^{\circ} \mathrm{C}$ (Mallard et al. 2018). Both temperature regimes have

208 the same daily temperature amplitude of $10^{\circ} \mathrm{C}$ (cold $10 / 20^{\circ} \mathrm{C}$ and hot $18 / 28^{\circ} \mathrm{C}$ ), but mean

209 temperatures differ $\left(15^{\circ} \mathrm{C}\right.$ in the cold and $23^{\circ} \mathrm{C}$ in the hot regime). The temperatures were chosen

210 such that one of the temperatures is stressful, whereas the other temperature is benign (David

211 1983; Petavy et al. 2001). Contrasting the founder population with hot-evolved generation 59,

212 Mallard et al. (2018) identified few (five) very pronounced selection peaks, some of them related

213 to metabolic alterations in the hot-evolved populations.

214 For an unbiased comparison of the two experiments, we added time series data for the hot-

215 evolved populations (F0, F15, F37, F59) and applied the same haplotype reconstruction pipeline

216 as described above. Similar to the cold-evolved population, the $\mathrm{X}$ chromosome had too few

217 outlier SNPs (114 SNPs) for haplotype reconstruction. 16 selected haplotype blocks were

218 identified on the two major autosomes (Supplementary Figure S3) and their selection

219 coefficients ranged from 0.05 to 0.13 (Figure 2B). It is remarkable that not only the number of

220 inferred selection targets, but also the distribution of selection coefficients is highly similar for

221 the two temperature regimes (hot-evolved $=16$ blocks, cold-evolved $=19$ blocks).

222 Only two haplotype blocks shared more SNPs than expected by chance between the two

223 temperature regimes. Furthermore, the shared region was in both cases only a small part of the

224 total haplotype block (Figure 1B and 1C, blocks 2 and 15). A prominent similarity between the

225 selection regimes could be identified in block 15, where the majority of overlapping SNPs were

226 located in the gene Ace (FBgn000024). Nevertheless, the selection pattern for Ace differs

227 between both temperature regimes (Langmüller et al. 2020). The shared SNPs of block 2 were

228 located in several genes, and therefore no clear candidate for common adaptation could be

229 identified in this region. 
230 We further scrutinized the haplotype blocks that were not shared between the selection regimes

231 and had a starting frequency higher than 0.15 to rule out that a selection signature in opposite

232 direction - as expected for a polygenic trait selected in contrasting environments - was missed.

233 The allele frequency change of all candidate SNPs in a haplotype block was always higher in the

234 focal temperature regime. Importantly, in both, hot and cold, selection regimes we very rarely

235 observed a frequency change in the opposite direction (Supplementary Figure S4). We conclude,

236 therefore, that we have no support for alleles being selected in opposite direction in hot and cold

237 temperatures. Rather, most alleles show a temperature-specific response.

238 Population-specific adaptation

239 Independent of the temperature regime the evolved populations derived from the Portugal

240 founder population revealed only a moderate number of selection targets. This contrasts a recent

241 experiment using the same hot temperature regime but a founder population from Florida

242 (Barghi et al. 2019). For a consistent comparison to the Portugal population, we repeated the

243 analysis of the Florida population using our haplotype reconstruction pipeline but focused only

244 on the two major autosomes. 31 selected haplotype blocks were identified on chromosome 2 and

2453 (Supplementary Figure S5), which are considerably fewer selected alleles than the 88 reported

246 by Barghi et al. (2019) for these two chromosomes. This difference reflects an alternative

247 strategy to identify candidate SNP sets for the haplotype reconstruction rather than the clustering

248 method (Otte and Schlötterer 2019). Following the same protocol as for the Portugal

249 experiments, 9,197 outlier SNPs were identified, which is more conservative than the 52,199

250 outlier SNPs used by Barghi et al. (2019) for haplotype reconstruction. The Florida population

251 harbored about twice as many selected haplotype blocks as the hot-evolved Portugal population.

252 For all experiments we identified the same relationship between starting frequency and selection

253 coefficients, but the distribution for Portugal was shifted towards higher selection coefficients

254 (Figure 2B). This result is robust with respect to the definition of selected alleles (see

255 Supplementary Figure S6). This difference in selection coefficients remains significant when we

256 account for allele frequencies in the founder populations (contrasts between estimated marginal

257 means Portugal cold - Florida hot $\mathrm{p}<0.0001$ and Portugal hot - Florida hot $\mathrm{p}<0.0001)$. No 
258 significant difference was observed between the two temperature regimes of the Portugal

259 population (Portugal cold - Portugal hot $\mathrm{p}=0.98$ ).

260 The Florida experiment was based on twice the number of replicates as the Portugal experiment.

261 To rule out that the number of replicates affects the inferred adaptive architecture, we repeated

262 the analysis with 100 sets of five randomly sampled Florida replicates. In all 100 random subsets

263 the selection coefficient was not different from the full data set (Wilcoxon rank-sum test $p>0.05$

264 adjusted for multiple testing using the Benjamini-Hochberg method), suggesting that the

265 difference between the Portugal and Florida data sets cannot be explained by a different number

266 of replicates.

267 We quantified the degree of parallelism between the experiments using the Jaccard index on the

268 selected haplotype blocks with $s$ significantly different from zero. High values indicate parallel

269 genetic responses whereas low values reflect heterogeneous, non-parallel genetic responses

270 between replicates. Jaccard indices were high for the two Portugal experiments (median

271 similarity between replicates $80 \%$ and $82 \%$, respectively). For the Florida experiment, the

272 Jaccard index was significantly lower (median similarity between replicates $70 \%$ for the full data

273 set; Wilcoxon rank-sum test p-value $<0.001$ against each Portugal data set), indicating less

274 parallel genetic responses and therefore increased realized genetic redundancy. This pattern was

275 robust with respect to the method used to define a selected allele in a given replicate (Figure 3

276 and Supplementary Figure S7). 
277 Figure 3: More parallel selection signatures among evolved replicate populations derived from Portugal

278 founders than from those with Florida ancestry. Jaccard indices comparing the different replicates in the 279 cold-evolved Portugal (top left), hot-evolved Portugal (top right) and hot-evolved Florida (bottom)

280 population. Jaccard index was computed based on estimation of significant selection coefficients (p-value $281<0.05)$.

282 The comparison of selected haplotype blocks between evolved populations derived from

283 different founder populations is difficult because the selected haplotype blocks are reconstructed

284 only for significant SNPs. Even if the selected haplotype block is shared, the remaining

285 haplotypes differ between the populations. As the frequency change at a given SNP on the

286 selected haplotype depends on the frequency of this SNP in the non-selected haplotypes,

287 different candidate SNPs are being identified - even if the same haplotype block is selected.

288 Thus, the same selected haplotype block may have different marker SNPs in two different

289 populations - suggesting that different haplotype blocks are selected. Furthermore, the low 
290 linkage disequilibrium in natural Drosophila populations (Charlesworth and Charlesworth 1973;

291 Langley et al. 1974) implies that very few haplotypes are expected to be shared between samples

292 from different populations. For these reasons, we did not attempt to test whether the same

293 haplotype blocks are selected in Portugal and Florida.

\section{Discussion}

295 D. simulans populations of different origin (Portugal and Florida) had very distinct adaptive

296 architectures in the same hot temperature regime (Mallard et al. 2018; Barghi et al. 2019):

297 Portugal had fewer selection targets, which were strongly selected in a highly parallel manner.

298 Florida harbored more selection targets with more heterogeneity among replicates and lower

299 selection coefficients compared to Portugal. In this report, we studied replicate populations

300 derived from the Portugal founder population, which adapted to a cold temperature regime to

301 understand why such different adaptive architectures were inferred in these two populations.

302 Below, we discuss several possible explanations for the differences in adaptive architecture.

\section{Different trait optima in the ancestral populations}

304 Both founder populations were collected on different locations with their own specific

305 temperature profile and in different phases of the seasonal cycle (Portugal in July 2008, Florida

306 in November 2010). Assuming that temperature adaptation is a single high-level trait, the

307 ancestral trait optimum may differ on the phenotypic axis. This implies that a population, which

308 is less adapted to hot environments should be better adapted to cold environments. Less well

309 adapted populations will experience stronger and more parallel selection responses across

310 replicates (Franssen et al. 2017b; Barghi et al. 2020), consistent with the pattern observed in the

311 hot Portugal population. Different trait optima of the founder populations were further supported

312 by the observation that the Portugal founder population is less fecund than the Florida founder

313 population when assayed in the novel hot environment (Barghi et al., unpublished results). This

314 implies that the mean phenotype of the Portugal population is more distant from the new trait

315 optimum in the hot laboratory environment than the Florida population. 
316 The analysis of the cold-evolved replicates casts some doubts on this simple interpretation. We

317 assumed that the hot and cold experiments shifted the trait optimum into opposite directions

318 relative to the (unknown) trait optimum of the ancestral Portugal population. Hence, contributing

319 alleles segregating at sufficiently high frequency in the ancestral population should be selected in

320 opposite direction in the two temperature regimes. Nevertheless, the results did not fit our

321 expectations - most selected haplotype blocks were not shared between the two temperature

322 regimes. While selected haplotype blocks starting from low frequencies may not be identified as

323 selection targets in the opposite temperature regime, also haplotype blocks with higher allele

324 frequencies in the founder population were not selected in opposite direction.

325 Hence, we conclude that temperature adaptation may not be a single high-level phenotype.

326 Rather, several sub-phenotypes on a lower level, which are not all shared for the different

327 temperature regimes, are contributing to adaptation. This conclusion is further supported by

328 different genomic signatures of hot and cold stressors in E\&R (Tobler et al. 2014) and QTL

329 mapping (Morgan and Mackay 2006) studies.

330 Differences in adaptive variation

331 Autosomal polymorphism levels differ between the two founder populations with Florida being

332 more variable than Portugal $\left(\pi_{\text {Florida }}=0.0076\right.$ and $\pi_{\text {Portugal }}=0.0062$, Wilcoxon rank-sum test on

333 non-overlapping $10 \mathrm{~kb}$ windows, $\mathrm{p}$-value $<0.001)$. Assuming that neutral variability is a good

334 approximation of adaptive variation, which is not always the case (Kellermann et al. 2009),

335 Portugal is expected to harbor less adaptive variation than Florida. This implies that Florida

336 reaches the trait optimum faster than Portugal (Thornton 2019; Barghi and Schlötterer 2020), but

337 in absence of phenotypic time series data, we cannot assess this hypothesis. A particularly

338 interesting hypothesis related to the different polymorphism levels is that Portugal harbors so

339 little adaptive variation that it does not have much genetic redundancy. This would imply that no

340 (or only limited) excess of adaptive genetic variation is segregating in the Portugal founder

341 population that can be used to reach the trait optimum. 
342 The Florida founder population, in contrast, harbors a considerable excess. Such differences in

343 the number of contributing loci can generate quite different patterns of parallel selection

344 responses (Barghi and Schlötterer 2020), matching the Portugal and Florida experiments.

345 Nevertheless, it is not apparent that the moderate differences in genome-wide polymorphism

346 levels are sufficiently large to explain this pattern.

$347 \quad$ Linkage disequilibrium

348 The above discussion about the heterogeneity of the inferred genetic architectures between

349 populations and selection regimes rests on the central assumption that the major contributing loci

350 were identified and could be distinguished with a recently developed haplotype reconstruction

351 approach (Otte and Schlötterer 2019). In other words, it is assumed that only a moderate number

352 of distinct loci contribute to adaptation.

353 Alternatively, the observed selection response may be explained by many loci of small effect -

354 an idea that matches in its extreme form the infinitesimal model (Barton et al., 2017). Empirical

355 support for a highly polygenic architecture of many traits comes from the strong correlation

356 between chromosome length and the fraction of heritability explained (Visscher et al. 2007;

357 Yang et al. 2011; Shi et al. 2016). If multiple small effect loci cluster together this may result in

358 a signature that will be interpreted as a single selection target (Yeaman and Whitlock 2011).

359 Short genomic segments with a local clustering of favored loci can even introgress and leave the

360 strong selection signature of a local allele frequency change (Sachdeva and Barton 2018).

361 Empirical support for the clustering of contributing loci comes from the molecular dissection of

362 candidate loci identified in QTL mapping studies. Single QTL loci can be broken into multiple

363 SNPs contributing to the corresponding trait (Stam and Laurie 1996; King et al. 2012; Kerdaffrec

364 et al. 2016; Gibert et al. 2017; Zan et al. 2017; Shahandeh and Turner 2020).

365 The situation in polymorphic founder populations is significantly more complicated than the

366 simple two genotype case studied by Sachdeva and Barton (2018), but we propose that blocks of

367 linked loci can not only generate pronounced selection signatures, but may also explain the

368 differences in adaptive architecture between the Portugal and Florida experiment. Depending on

369 the extent of linkage disequilibrium (LD) the clustering of contributing loci can vary. Hence, 
370 populations with different levels of LD may also harbor more or less clustered contributing loci.

371 The influence of haplotype structure can be illustrated by two extreme cases: in the case of 372 complete linkage equilibrium (LE), in any genomic window the haplotypes segregating in the

373 population should have similar fitness despite being highly diverse. As a consequence, changes

374 in trait optimum will result only in rather small frequency changes of the haplotypes in this

375 genomic window. This pattern becomes more pronounced with an increasing number of

376 contributing loci.

377 On the other hand, in the presence of strong linkage disequilibrium, fewer distinct haplotypes are 378 present in a given genomic window. Sampling variation in the ancestral population generates

379 haplotypes with different numbers of contributing loci in a given genomic window. The more

380 pronounced the difference in the number of loci among haplotypes in a genomic window is, the

381 stronger will be the fitness differences among them and thus the allele frequency change after a

382 shift in trait optimum. Hence, because the difference in the number of contributing loci among

383 haplotypes differs among genomic windows, linkage disequilibrium generates heterogeneity in

384 selection response along the chromosome.

385 We illustrated the impact of LD by assuming about 1200 contributing loci genome-wide and

386 simulated a window size of $1 \mathrm{Mb}$ in a typical E\&R setting. For high LD and LE the same

387 number of chromosomes with beneficial alleles was used. 100 different genomic windows were

388 simulated and simulations with higher LD resulted in a more heterogeneous response to

389 selection. Consistent with a larger phenotypic variance (Figure 4A) also much more pronounced

390 allele frequency changes were observed for some windows in the presence of linkage

391 disequilibrium (Figure 4B). Hence, while very homogeneous moderate frequency shifts were

392 observed for beneficial alleles in linkage equilibrium, some pronounced sweep windows were

393 detected for windows with LD. We also assessed the degree of parallelism in the response

394 between replicates and found a more parallel response for the high LD simulations (Figure 4C). 

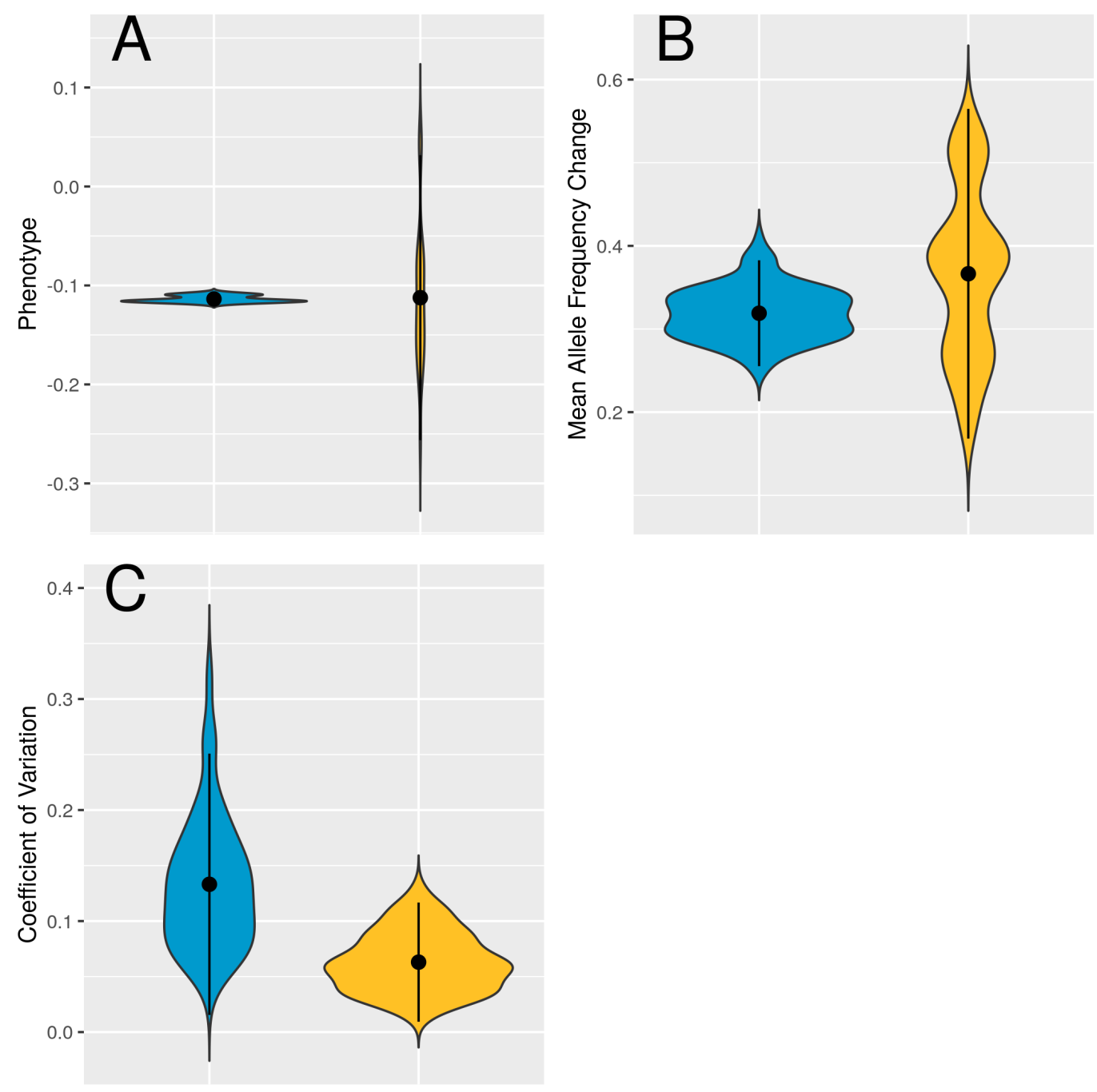

Figure 4: Influence of linkage disequilibrium on the genomic response of a polygenic trait. We simulated 50 generations of polygenic adaptation after a shift in trait optimum. 100 replicates, each with 10 loci in a $1 \mathrm{Mb}$ region either with linkage equilibrium (blue) or strong LD (yellow) are shown in violin plots. A) Independent of linkage structure, the same mean phenotype was reached, but simulations with high LD were considerably more scattered. B) Pronounced allele frequency changes were obtained for both, linkage equilibrium und high LD. For linkage equilibrium, the genomic windows were all rather similar, indicating that no window showed a strong selection signature distinguishing it from the genomic background. Simulations with strong LD, however, resulted in highly heterogeneous selection responses, with some windows having a frequency change larger than 0.6 , which is a strong selection signature distinguishing it from the remaining genomic windows. C) The heterogeneity among five replicate populations is measured by the coefficient of variation. The selection response in simulations with linkage equilibrium was less parallel than in those with strong LD. 
407 Thus, for polygenic traits the inferred adaptive architecture can be strongly affected by linkage in

408 the ancestral population. A previous study on the impact of recombination on the response to a

409 shift in trait optimum with truncating selection observed more heterogeneity among replicates in

410 the case of linkage equilibrium than for complete linkage (Zhang and Hill 2005). We attribute

411 these differences to the small population sizes in Zhang and Hill (2005).

412 Nevertheless, does this scenario of a highly polygenic architecture with differences in LD apply

413 to the Florida and Portugal experiments? Following the same rationale as Shi et al. (2016),

414 Barghi et al. (2019) tested whether longer haplotype blocks were more strongly selected than

415 shorter ones, but no significant correlation was found. Similar results were observed for the cold

416 evolved Portugal populations. We caution, however, that these negative results do not provide

417 strong support for the identification of distinct selection targets. Possible, not mutually exclusive,

418 reasons for the lack of significance even in the presence of a highly polygenic architecture are:

419 1) the contributing loci have different effect sizes, thus small haplotype blocks with large effect

420 loci may be more strongly selected ones than larger blocks, 2) larger blocks may harbor more

421 loci with effects in opposite direction than smaller ones, 3) since haplotype blocks are still

422 relatively short (compared to full chromosomes) stochastic sampling and heterogeneity in the

423 density of contributing loci may obscure the correlation between size of the selected haplotype

424 block and the selection response.

425 The hypothesis that LD differences can explain the heterogeneity in inferred adaptive

426 architecture is supported by the observation that the two founder populations differed across all

427 chromosomes in their pattern of linkage disequilibrium (Figure 5). The higher LD in Portugal

428 compared to Florida is fully consistent with the prediction that founder populations with higher

429 LD are more likely to show stronger and more parallel sweep signatures than populations with

430 lower LD. Although this pattern fits our observations, in absence of more information about the

431 degree of polygenicity and the distribution of contributing loci across the chromosomes and their

432 effect sizes, it is not possible to determine whether the observed differences in LD are sufficient

433 to explain our empirical data. 

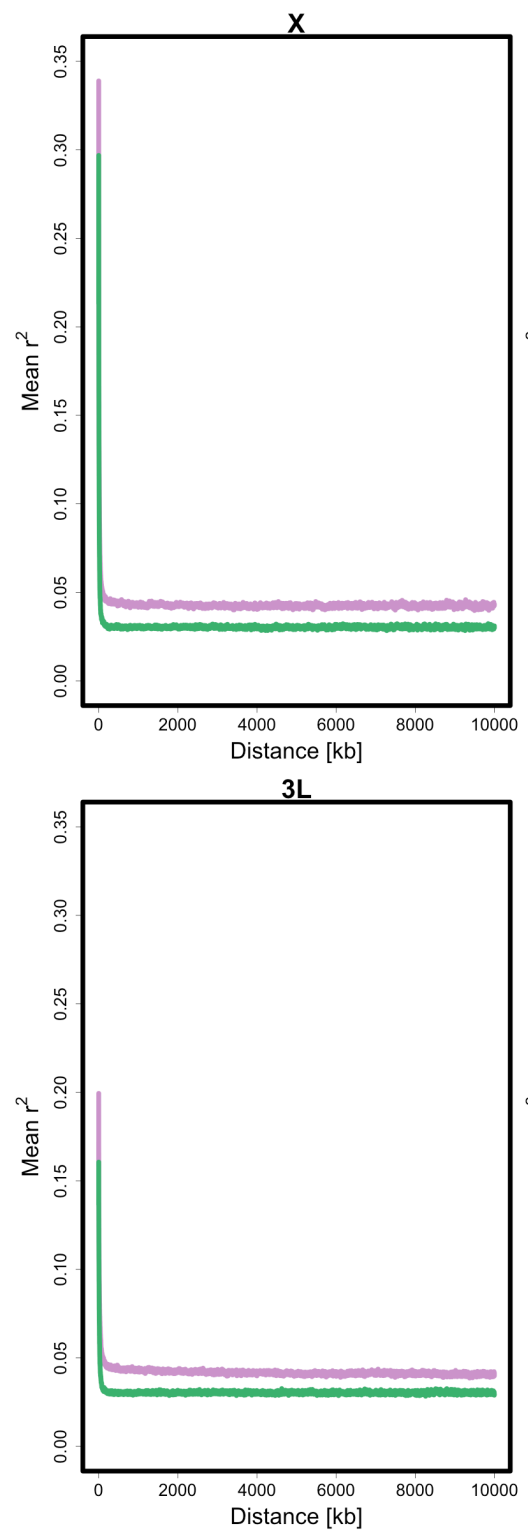
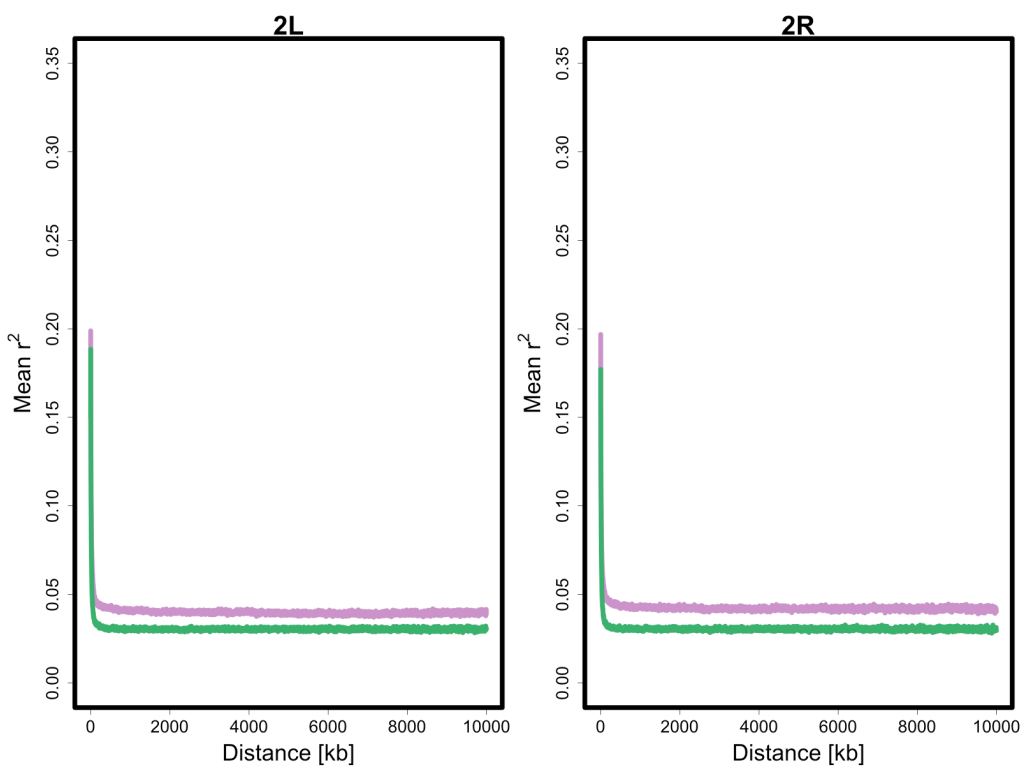

$3 R$

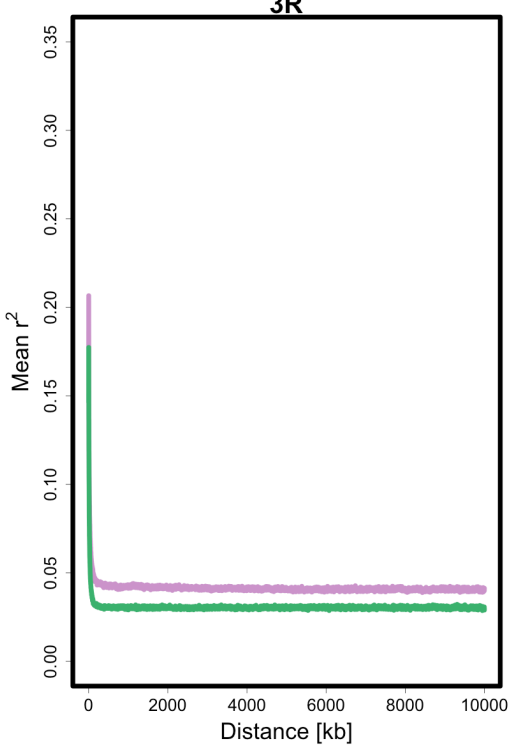

434 Figure 5: Linkage disequilibrium in the ancestral Portugal und Florida population as measured by the mean $\mathrm{r}^{2}$ of loci with distances up to $10,000 \mathrm{~kb}$ based on 34 individual haplotype sequences. 
436 Temperature adaptation may involve multiple, temperature-specific traits

437 While we cannot pinpoint the cause for the differences in the inferred adaptive architecture

438 between Portugal and Florida, the analysis of cold-evolved replicates shed some important light

439 on our understanding of temperature adaptation.

440 Many studies, in particular theoretical ones, considered high level phenotypes, such as

441 temperature adaptation, as a single adaptive trait, where shifts in mean temperature are treated as

442 a simple shift in trait optimum (e. g. Bridle et al. 2009; Chevin et al. 2010; Hoffmann 2010;

443 Kopp and Matuszewski 2014). This implies that all segregating contributing loci affect the

444 optimal phenotype - irrespective of the position of the optimum - i.e. in the case of temperature

445 adaptation irrespective of whether the optimum is in the hot or cold. The limited overlap between

446 the selection targets in hot- and cold-evolved replicates is striking, as it contradicts this

447 assumption. With the exception of the genomic region around the Ace locus and a region across

448 the centromere of chromosome 2, which changed in the same direction in both temperature

449 regimes, no shared haplotype blocks were detected. To some extent, the lack of shared haplotype

450 blocks can be attributed to low starting frequencies, which implies that selection in the opposite

451 direction does not result in allele frequency changes sufficiently large to be detected.

452 Nevertheless, even for haplotype blocks starting from intermediate frequency, no selection

453 signature in the opposite direction was noticed.

454 This implies that different loci are contributing to adaptation in hot and cold environments -

455 irrespective of whether a highly or moderately polygenic architecture is assumed. A very similar

456 lack of shared candidates was also noticed in a D. melanogaster experiment, where replicate

457 populations were exposed to the same hot and cold temperature regimes (Tobler et al. 2014).

458 This experiment was, however, conducted for a much smaller number of generations, and the

459 selection signature was analyzed on the SNP-level, which makes the interpretation of the results

460 particularly challenging given the contribution of large segregating inversions to temperature

461 adaptation in this species (Hoffmann et al. 2002; Rako et al. 2007).

462 The observation of different selection targets in hot and cold environments is particularly

463 interesting, because seasonal changes were found to be associated with cycling allele frequencies

464 in natural D. melanogaster populations (Bergland et al. 2014), which suggests that the same 
465 SNPs are being selected in opposite direction in hot and cold environments. One possible

466 explanation for these differences to our study is that in natural populations only a moderate

467 number of generations separates the two temperature regimes, while in our experiment the

468 temperature regime remained constant across more than 50 generations.

469 Population-specific adaptive architectures

470 Various studies, mainly using QTL mapping and GWAS, have identified different loci

471 contributing to the same trait in diverged populations (Adeyemo et al. 2009; Wu et al. 2013; Al

472 Olama et al. 2014; Li and Keating 2014; Conte et al. 2015; Horikoshi et al. 2018; Takata et al.

473 2019; Wojcik et al. 2019; Zan and Carlborg 2019; Hodonsky et al. 2020; Kemppainen et al.

474 2020). A recent experimental evolution study using D. subobscura populations with different

475 genetic background also observed very little overlap in the genomic regions responding to a

476 common selection regime (Seabra et al. 2018). Hence, the different selection targets obtained

477 from Portugal and Florida experiments conducted in the same hot environment are not

478 particularly surprising and emphasize the limited insights about the genetic basis of a polygenic

479 trait from single population studies. Very surprising, however, was the observation that the

480 adaptive architecture (number of contributing loci, effect sizes and starting frequencies) was

481 different between Portugal and Florida, but strikingly similar between the hot and cold selection

482 regime.

483 Although more experiments are needed to nail down why the adaptive architecture is highly

484 dependent on the founder population and not on the selection regime, our results have important

485 implications for all studies attempting to characterize the adaptive architecture. The analysis of a

486 single population cannot be sufficient to understand the genetic basis of adaptive traits. Thus,

487 multiple diverged populations need to be studied to reach conclusions that can be generalized

488 beyond a limited number of focal populations. 


\section{Material and Methods}

490 Unless stated otherwise, analysis was conducted using R v3.6.1 (R Core Team 2019).

\section{Experimental Populations and Selection Regime}

492 The set-up of the evolution experiment is described in detail elsewhere (Mallard et al. 2018). In

493 brief, female flies were sampled from a natural Drosophila simulans population in Northern

494 Portugal in the summer of 2008 and used to establish 250 isofemale lines. These lines were kept

495 for 10 generations in the laboratory before starting the experiment. Mated females of all lines

496 were used to create the starting populations. Ten replicates were created by combining an equal

497 number of flies from each line. Five of the replicates were then kept in a $12 \mathrm{~h}: 12 \mathrm{~h}$ day and night

498 cycle with temperatures of $20^{\circ} \mathrm{C}$ during the day and $10^{\circ} \mathrm{C}$ during the night (cold regime). The

499 population size was kept constant at 1250 per replicate in non-overlapping generations.

500 The other five replicates were used to start the evolution experiment described by Mallard et al.

501 (2018), which was identical to the cold regime except for the temperature which fluctuated

502 between $28^{\circ} \mathrm{C}$ during the day and $18^{\circ} \mathrm{C}$ at night (hot regime).

\section{$503 \quad$ Evolve \& Resequence}

504 For the founder population, we used the sequences described by Mallard et al. (2018), but added

505 new sequence data from two replicates of generation F3 from the cold regime to increase

506 coverage. To avoid biases related to different sequencing approaches, all reads were pooled and

507 then randomly split into five subsets with a coverage of 100x each. These subsets were used as

508 founder population replicates throughout the analysis of the cold-evolved and the (re-)analysis of

509 the hot-evolved populations. While sequence data for the F59 in the hot regime were available

510 from Mallard et al. (2018), we added new Pool-Seq data for the intermediate time points F15 and

511 F37 in the hot regime to allow for the time series analysis and haplotype reconstruction in this

512 study. All sequences used for the founder population (including the F3 from the cold regime) and

513 all sequences of the hot regime were derived from females only. 
514 Sequencing data from the time points F11, F21, F31, F41 and F51 in the cold regime were newly

515 generated from pooled females and males. Paired-end libraries were generated with different

516 protocols and sequenced on different Illumina platforms (see Supplementary Table S1).

\section{Data processing}

518 Sequencing reads were trimmed using Readtools TrimFastq version 0.2. (Gomez-Sanchez and

519 Schlötterer 2017) with the parameters --minReadLength 50 --disable5pTrim --

520 mottQualityThreshold 20. The D. simulans genome sequence created by Palmieri et al. (2015)

521 was used as reference for read mapping. To avoid false positive outlier SNPs, which might arise

522 when libraries with different read lengths and insert sizes are combined in one analysis (Kofler et

523 al. 2016), three different mappers were used to map reads of the two time points used for outlier

524 testing (cold regime: F0 and F51; hot regime: F0 and F59). Reads were mapped using Bowtie2

525 version 2.2.6 (Langmead and Salzberg 2012) with parameters --end-to-end --X 1500, bwa mem

526 version 0.7.13 (Li and Durbin 2010) with default parameters and novoalign version 3.03.2

527 (Novocraft 2014) with parameters -i 350,100 -F STDFQ -o SAM -r RANDOM.

528 The intermediate time points (cold regime: F11, F21, F31, F41; hot regime F15, F37) used for

529 the detection of selected haplotype blocks were mapped with novoalign only, as this mapper is

530 known to estimate allele frequencies most accurately (Kofler et al. 2016).

531 Mapped reads were filtered for mapping quality $\geq 20$ and proper pairs using SAMtools view

532 version 1.3.1 ( $\mathrm{Li}$ et al. 2009). Duplicates were removed using picard MarkDuplicates version

533 2.1.1 (Broad Institute 2019). Barcoded files were split using Readtools

534 AssignReadGroupByBarcode version 0.2.2 (Gomez-Sanchez and Schlötterer 2017) with

535 parameters --maximumMismatches 1. The BAM files were then used to create mpileup files with

536 SAMtools mpileup version 1.3.1 (Li et al. 2009), and finally, PoPoolation2 mpileup2sync.jar

537 version 1.201 (Kofler et al. 2011) was used to create sync files from mpileup files. All

538 subsequent analysis was conducted on the basis of these sync files. 
540 Single nucleotide polymorphisms (SNPs) were called from the founder population by creating

541 sync files from BAM files as described above but filtering for polymorphic sites that had a

542 mapping quality of at least 30 and a minimal count of at least 5 and were detected by the three

543 mapping algorithms in all founder replicates. Filtering resulted in 3.8 million SNPs that were

544 used for further analysis. Indels were detected using the PoPoolation2 identify-indel-regions.pl

545 script, and transposable elements were detected with repeatmasker v 1.332 (Smit et al. 2015).

546 Custom databases made by combining RepeatMasker database Dfam_Consensus-20181026,

547 RepBase-20181026, and transposon_sequence_set.embl.txt from flybase.org (FB 2018_06), and

548 search engine NCBI/RMBLAST v 2.2.27+ were used for repeats $>500 \mathrm{bp}$. All sync files were

549 masked for these repetitive regions and for known Y chromosome translocations (Tobler et al.

550 2017) using the PoPoolation2 filter-sync-by-gtf.pl script.

$551 \quad$ Correcting for different insert sizes

552 To correct for false positive outlier SNPs created by libraries with different insert sizes (Kofler et

553 al. 2016), mapping results from the three different mappers for the founder (F0) and most

554 evolved (cold regime: F51; hot regime F59) population were used. $\chi^{2}$ tests were conducted to

555 compare the results of the different mappers per replicate and time point. After correcting for

556 multiple testing using the Benjamini-Hochberg procedure (Benjamini and Hochberg 1995), only

557 SNPs that showed a consistent response across comparisons (p.adjust $\geq 0.05$ ) were kept for

558 further analysis.

\section{Candidate SNPs}

560 Candidate outlier SNPs were detected in the filtered sync files created from novoalign mapping

561 results after correcting for false positive outliers as described above. Allele frequency changes

562 between the founder and the most evolved population were analyzed using $\mathrm{CMH}$ and $\chi^{2}$ tests

563 which are adapted for genetic drift and pool sequencing noise as implemented in the R package

564 ACER version 1.0 (Spitzer et al. 2020). SNPs within the top 1\% of coverage were excluded from 
565 the analysis to avoid copy number variants. Intermediate generations were included in the

566 correction approach. Effective population size per replicate was calculated using the R package

567 poolSeq version 0.3.5 (Taus et al. 2017) with the function estimateWndNe (window size $10 \mathrm{~kb}$,

568 method P.planI, pool size and census size 1250) and used for CMH and $\chi^{2}$ tests. The CMH test

569 was performed using all population replicates per time point whereas $\chi^{2}$ tests were performed for

570 each replicate separately. All results were corrected for multiple testing (Benjamini-Hochberg).

571 Finally, candidate SNPs detected by either test (p.adjust $<0.05$ ) were combined to include

572 consistent responses across replicates (CMH test) and replicate-specific responses $\left(\chi^{2}\right.$ test).

\section{Selected haplotype blocks}

574 Selected haplotype blocks were reconstructed from candidate SNP allele frequency data of all

575 time points and replicates using the R package haplovalidate with MNCS of 0.01 (Otte and

576 Schlötterer 2019). Haplotype blocks were reconstructed for all time points. As haplotype blocks

577 might contain more than one selected allele, early time points (cold regime: F11, F21 and F31;

578 hot regime: F15 and F37) were used for fine-mapping of selected haplotype blocks (Otte and

579 Schlötterer 2019). Here, the analysis detected the characteristic signal of reconstructed haplotype

580 blocks with multiple selection targets, which is the presence of a single haplotype block in the

581 most evolved generation but several reconstructed haplotype blocks when analyzing the early

582 generations separately. Haplotype blocks from the early generations showing this pattern were

583 included in the final analysis.

584 Selection coefficients for the detected haplotype blocks per replicate were computed using the

585 allele frequency trajectories of the top $10 \%$ outlier SNPs based on CMH and $\chi^{2}$ test result and

586 using the poolSeq v0.3.5 function estimateSH (method $L L S$ ) (Taus et al. 2017). Only selection

587 coefficients with p-value $<0.05$ were used to calculate the median selection coefficient for each

588 selected allele. Relationship of selection coefficient, starting allele frequency and block size were

589 tested using a linear model with $\log 10$ transformation of selection coefficients. To test the

590 robustness of our definition for selected alleles, we repeated the estimation of selection

591 coefficients using a) the top 20\% SNPs or b) all SNPs that had an allele frequency change $>0.1$

592 (Supplementary Figure S1). Candidate genes per block were detected from the gene annotation 
593 of the reference genome (Palmieri et al. 2015) including also SNPs 200 bp up- and downstream

594 of the focal gene.

595 Comparison to other experimentally evolved $D$. simulans populations

596 We used data from the same $D$. simulans population evolving under a hot temperature regime

597 (Mallard et al. 2018) to contrast adaptation to different temperatures. For this population, we

598 included two additional time points, so that four time points in total were available: F0, F15,

599 F37, F59. The data set was filtered, candidate SNPs were detected and haplotypes were

600 reconstructed in the same way as described above including the combined analysis of all (F0-

601 F59) and early (F0-F37) time points. To estimate how many shared marker SNPs were expected

602 by chance, we randomly sampled the number of shared SNPs from the haplotype blocks,

603 calculated the fraction per haplotype block $(\mathrm{N}=10,000)$ and finally applied a $95 \%$ cut-off.

604 In addition, a different published hot-evolved D. simulans population from Florida (Barghi et al.

605 2019) was used for the comparative analysis. The data set was filtered and candidate SNPs were

606 detected in the same way as described above. As intermediate time points were available for this

607 data set (every 10th generation from F0 to F60), haplovalidate (Otte and Schlötterer, 2019) with

608 MNCS of 0.01 was used to detect selected haplotype blocks including the combined analysis of

609 all (F0-F60) and early (F0-F30) time points as described for the Portugal population. Selection

610 coefficients were computed as described above.

611 We fitted a linear model with $\log 10$ transformed selection coefficients as response and main

612 effects of population as fixed categorical effect with three levels (Florida hot, Portugal hot,

613 Portugal cold) and a linear and quadratic covariate for starting allele frequency, to account for

614 their non-linear relationship with the response, as explanatory variables. Residuals from this

615 model were normally distributed and displayed variance homogeneity. The model with linear

616 and quadratic covariate for starting allele frequency fit significantly better than a model with

617 only a linear term. Contrasts between populations were compared based on estimated marginal

618 means (R package emmeans).

619 We compared the similarity of replicates calculating the Jaccard indices for the Portugal and the

620 Florida population using the R package philentropy (Drost 2018). We created binary data based 
621 on the replicate-specific selection response, i.e. whether or not a significant selection coefficient

622 could be estimated by the poolSeq package (see above) for the corresponding replicate and allele

623 (p-value <0.05). Following Barghi et al. (2019), we created binary data by applying a cut-off of

6240.1 to the median allele frequency change of selected alleles per replicate. Jaccard indices

625 between populations were compared using the two-sample Wilcoxon rank-sum test. To analyze

626 the effect of the different number of replicates (10 in the Florida and five in the Portugal

627 population) we repeated the analysis of selection coefficients and Jaccard indices on a

628 downsampled set of Florida replicates. For this analysis, we took 100 random samples of five

629 replicates from the Florida population data set and repeated the analysis for each of them as

630 described above.

\section{Nucleotide diversity in the ancestral populations}

632 Nucleotide diversity $(\pi)$ of each autosome in the ancestral populations was calculated from the

633 allele frequency data using the formula of Tajima (1989). For maintaining a comparable number

634 of low-frequency alleles we subsampled the Florida data set to five replicates. As different sets

635 of five Florida replicates resulted in very consistent $\pi$ estimates (data not shown) we only used

636 one set for the direct comparison to Portugal.

637 Linkage disequilibrium in the ancestral populations

638 To quantify linkage disequilibrium, we used 189 haplotype sequences of the Florida founder

639 population (Howie et al. 2019) and 34 haplotype sequences of the Portugal founder population

640 which are described in Langmüller et al. (2020). For maintaining a comparable number of low-

641 frequency alleles, we subsampled the Florida data set to 34 haplotypes. We calculated the mean

$642 \mathrm{r}^{2}$ for loci (minor allele count $=3$, minimum SNP quality $=50$ ) within $10,000 \mathrm{~kb}$ distance. As

643 different sets of 34 Florida haplotypes resulted in very consistent mean estimates (see

644 Supplementary Figure S8) we only used one set for the direct comparison to Portugal. 
646 We illustrated the effect of linkage equilibrium (LE) or strong linkage disequilibrium (LD) on

647 polygenic adaptation after a shift in trait optimum with computer simulations using MimicrEE2

648 v208 (Vlachos and Kofler 2018) in qff mode. We used parameters that matched the Portugal $D$.

649 simulans E\&R experiment with five replicates, each starting with the same 1000 homozygous

650 individuals which evolved for 50 generations. For computational simplicity, we assumed that all

65110 loci contributing to the phenotype, each with a starting allele frequency of 0.05 and effect size

652 of 0.05 , are restricted to a $1 \mathrm{Mb}$ region. 100 independent simulations were performed to mimic

653100 different genomic regions. We used a Gaussian fitness function as previously described (e.g.

654 Barghi and Schlötterer 2020): minimum fitness 0.5, maximum fitness 4.5, standard deviation of

655 the phenotype 1.2 , heritability of 0.5 . The mean fitness of the ancestral population was -0.44 and

656 the new trait optimum was 0.5 . We used the average recombination rate of $D$. simulans

657 (Dsim_recombination_map_LOESS_100kb_1.txt, (Howie et al. 2019)). We generated two

658 different sets of founder populations, one with strong LD and one with linkage equilibrium (LE).

659 Both sets of founder populations contained 200 chromosomes with favored alleles and 800

660 chromosomes without. For strong LD, four different sets of selected haplotypes were generated,

661 and the number of contributing loci was randomly distributed between the four sets of selected

662 haplotypes. Hence, 50 haplotypes had the same number of contributing loci, but due to stochastic

663 sampling the number of contributing loci differs among the four sets of 50 haplotypes. To

664 generate starting populations in LE, we randomly distributed the selected alleles across 200

665 haplotypes until each of the alleles had a final frequency of 0.05.

666 After 50 generations, we generated "Pool-seq data" with 50x coverage and added sequencing

667 noise by binomial sampling based on the allele frequencies. We recorded the phenotypic and

668 mean frequency change across loci and replicates as well as the coefficient of variation in the

669 mean allele frequency change across the five replicates as an indicator for the degree of parallel

670 response. 


\section{Data Availability}

672 Sequence data were deposited at the European Nucleotide Archive (ENA) under the accession

673 number XXX. Population sync files, all results and scripts were deposited on Dryad Digital

674 Repository XXX.

\section{Acknowledgments}

676 We thank Neda Barghi for sharing unpublished fecundity data and for providing information

677 concerning the hot-evolved D. simulans data set. Anna Langmüller, Thomas Taus and Claire

678 Burny shared code to remove SNPs with allele frequency estimates that are sensitive to the insert

679 sizes of NGS sequencing libraries. The authors also thank the members of the Institute of

680 Population Genetics for discussion and support on the project. Special thanks to Marlies Dolezal

681 for in depth statistical advice. KAO was supported by a DFG Research Fellowship (OT 532/1-1).

682 FM was supported by a Marie Sklodowska-Curie Individual Fellowship (H2020-MSCA-IF-

683 661149). CS was supported by the European Research Council grant "ArchAdapt" and the

684 Austrian Science Funds (FWF, P27630, P29133). Illumina sequencing for a subset of the data

685 was performed at the VBCF NGS Unit (www.viennabiocenter.org/facilities). 


\section{References}

Adeyemo A, Gerry N, Chen G, Herbert A, Doumatey A, Huang H, Zhou J, Lashley K, Chen Y, Christman $\mathrm{M}$ et al. 2009. A genome-wide association study of hypertension and blood pressure in African Americans. PLoS Genet 5: e1000564.

Al Olama AA Kote-Jarai Z Berndt SI Conti DV Schumacher F Han Y Benlloch S Hazelett DJ Wang Z Saunders E et al. 2014. A meta-analysis of 87,040 individuals identifies 23 new susceptibility loci for prostate cancer. Nat Genet 46: 1103-1109.

Barghi N, Hermisson J, Schlötterer C. 2020. Polygenic adaptation: a unifying framework to understand positive selection. Nature reviews Genetics: in press.

Barghi N, Schlötterer C. 2020. Distinct patterns of selective sweep and polygenic adaptation in evolve and re-sequence studies. Genome biology and evolution doi:10.1093/gbe/evaa073.

Barghi N, Tobler R, Nolte V, Jaksic AM, Mallard F, Otte KA, Dolezal M, Taus T, Kofler R, Schlötterer C. 2019. Genetic redundancy fuels polygenic adaptation in Drosophila. PLoS biology 17: e3000128.

Barton NH, Etheridge AM, Veber A. 2017. The infinitesimal model: Definition, derivation, and implications. Theoretical population biology 118: 50-73.

Barton NH, Keightley PD. 2002. Understanding quantitative genetic variation. Nature reviews Genetics 3: 11-21.

Bataille L, Delon I, Da Ponte JP, Brown NH, Jagla K. 2010. Downstream of identity genes: muscle-type-specific regulation of the fusion process. Dev Cell 19: 317-328.

Beavis WD. 1998. QTL analyses: power, precision, and accuracy.

Benjamini Y, Hochberg Y. 1995. Controlling the false discovery rate: a practical and powerful approach to multiple testing. . J R Stat Soc Ser 57: 289-300.

Bergland AO, Behrman EL, O'Brien KR, Schmidt PS, Petrov DA. 2014. Genomic evidence of rapid and stable adaptive oscillations over seasonal time scales in Drosophila. PLoS genetics 10: e1004775.

Bridle JR, Gavaz S, Kennington WJ. 2009. Testing limits to adaptation along altitudinal gradients in rainforest Drosophila. Proceedings Biological sciences / The Royal Society 276: $1507-1515$.

Broad Institute. 2019. Picard toolkit. http://broadinstitute.github.io/picard/.

Bulmer MG. 1971. Effect of Selection on Genetic Variability. Am Nat 105: 201-\&.

Charlesworth B, Charlesworth D. 1973. A study of linkage disequilibrium in populations of Drosophila melanogaster. Genetics 73: 351-359.

Chevin LM, Lande R, Mace GM. 2010. Adaptation, plasticity, and extinction in a changing environment: towards a predictive theory. PLoS biology 8: e1000357.

Conte GL, Arnegard ME, Best J, Chan YF, Jones FC, Kingsley DM, Schluter D, Peichel CL. 2015. Extent of QTL Reuse During Repeated Phenotypic Divergence of Sympatric Threespine Stickleback. Genetics 201: 1189-1200.

David JR, Allemand, R., Van Herrewege, J. and Cohet, Y. . 1983. Ecophysiology: abiotic factors. In The Genetics and Biology of Drosophila, Vol 3d (ed. M Ashburner, Carson, H. L. \& Thompson, J. N. Jr ), pp. 105-170. Academic Press, London. 
de Vladar HP, Barton N. 2014. Stability and response of polygenic traits to stabilizing selection and mutation. Genetics 197: 749-767.

Drost HG. 2018. Philentropy: Information Theory and Distance Quantification with R. Journal of Open Source Software 3: 765.

Eyre-Walker A. 2010. Evolution in health and medicine Sackler colloquium: Genetic architecture of a complex trait and its implications for fitness and genome-wide association studies. Proc Natl Acad Sci U S A 107 Suppl 1: 1752-1756.

Field Y, Boyle EA, Telis N, Gao Z, Gaulton KJ, Golan D, Yengo L, Rocheleau G, Froguel P, McCarthy MI et al. 2016. Detection of human adaptation during the past 2000 years. Science 354: 760-764.

Fisher RA. 1918. The correlation between relatives on the supposition of Mendelian Inheritance. Trans Roy Soc Edinburgh 52: 339-433.

Flint J, Mott R. 2001. Finding the molecular basis of quantitative traits: successes and pitfalls. Nat Rev Genet 2: 437-445.

Franssen SU, Barton NH, Schlötterer C. 2017a. Reconstruction of haplotype-blocks selected during experimental evolution. Molecular biology and evolution 118: 42-51.

Franssen SU, Kofler R, Schlötterer C. 2017b. Uncovering the genetic signature of quantitative trait evolution with replicated time series data. Heredity 118: 42-51.

Garland T, Rose MR. 2009. Experimental Evolution: concepts, methods, and applications of selection experiments. University of California Press, Berkeley.

Gazal S, Finucane HK, Furlotte NA, Loh PR, Palamara PF, Liu X, Schoech A, Bulik-Sullivan B, Neale BM, Gusev A et al. 2017. Linkage disequilibrium-dependent architecture of human complex traits shows action of negative selection. Nature genetics 49: 1421-1427.

Gibert JM, Blanco J, Dolezal M, Nolte V, Peronnet F, Schlötterer C. 2017. Strong epistatic and additive effects of linked candidate SNPs for Drosophila pigmentation have implications for analysis of genome-wide association studies results. Genome biology 18: 126.

Gomez-Sanchez D, Schlötterer C. 2017. ReadTools: A universal toolkit for handling sequence data from different sequencing platforms. Molecular ecology resources doi:10.1111/1755-0998.12741.

Harris AM, DeGiorgio M. 2020. Identifying and Classifying Shared Selective Sweeps from Multilocus Data. Genetics 215: 143-171.

Hodonsky CJ, Baldassari AR, Bien SA, Raffield LM, Highland HM, Sitlani CM, Wojcik GL, Tao R, Graff M, Tang W et al. 2020. Ancestry-specific associations identified in genomewide combined-phenotype study of red blood cell traits emphasize benefits of diversity in genomics. BMC Genomics 21: 228.

Hoffmann AA. 2010. Physiological climatic limits in Drosophila: patterns and implications. $J$ Exp Biol 213: 870-880.

Hoffmann AA, Anderson A, Hallas R. 2002. Opposing clines for high and low temperature resistance in Drosophila melanogaster. Ecol Lett 5: 614-618.

Höllinger I, Pennings PS, Hermisson J. 2019. Polygenic adaptation: From sweeps to subtle frequency shifts. PLoS genetics 15: e1008035. 
Horikoshi M, Day FR, Akiyama M, Hirata M, Kamatani Y, Matsuda K, Ishigaki K, Kanai M, Wright H, Toro CA et al. 2018. Elucidating the genetic architecture of reproductive ageing in the Japanese population. Nat Commun 9: 1977.

Howie JM, Mazzucco R, Taus T, Nolte V, Schlotterer C. 2019. DNA motifs are not general predictors of recombination in two Drosophila sister species. Genome biology and evolution doi:10.1093/gbe/evz082.

Jain K, Stephan W. 2017a. Modes of rapid polygenic adaptation. Molecular biology and evolution 34: 3169-3175.

Jain K, Stephan W. 2017b. Rapid Adaptation of a Polygenic Trait After a Sudden Environmental Shift. Genetics 206: 389-406.

Jensen JD, Kim Y, DuMont VB, Aquadro CF, Bustamante CD. 2005. Distinguishing between selective sweeps and demography using DNA polymorphism data. Genetics 170: 14011410.

Jha AR, Miles CM, Lippert NR, Brown CD, White KP, Kreitman M. 2015. Whole-genome resequencing of experimental populations reveals polygenic basis of egg-size variation in Drosophila melanogaster. Molecular biology and evolution 32: 2616-2632.

Jha AR, Zhou D, Brown CD, Kreitman M, Haddad GG, White KP. 2016. Shared Genetic Signals of Hypoxia Adaptation in Drosophila and in High-Altitude Human Populations. Mol Biol Evol 33: 501-517.

Johnson KE, Voight BF. 2018. Patterns of shared signatures of recent positive selection across human populations. Nat Ecol Evol 2: 713-720.

Johri P, Charlesworth B, Jensen JD. 2020. Toward an Evolutionarily Appropriate Null Model: Jointly Inferring Demography and Purifying Selection. Genetics 215: 173-192.

Jones FC, Grabherr MG, Chan YF, Russell P, Mauceli E, Johnson J, Swofford R, Pirun M, Zody $\mathrm{MC}$, White $\mathrm{S}$ et al. 2012. The genomic basis of adaptive evolution in threespine sticklebacks. Nature 484: 55-61.

Kawecki TJ, Lenski RE, Ebert D, Hollis B, Olivieri I, Whitlock MC. 2012. Experimental evolution. Trends in ecology \& evolution 27: 547-560.

Kellermann V, van Heerwaarden B, Sgro CM, Hoffmann AA. 2009. Fundamental evolutionary limits in ecological traits drive Drosophila species distributions. Science 325: 1244-1246.

Kelly JK, Hughes KA. 2019. Pervasive Linked Selection and Intermediate-Frequency Alleles Are Implicated in an Evolve-and-Resequencing Experiment of Drosophila simulans. Genetics 211: 943-961.

Kemppainen P, Li Z, Rastas P, Löytynoja A, Fang B, Guo B, Shikano T, Yang J, Merilä J. 2020. Genetic population structure constrains local adaptation and probability of parallel evolution in sticklebacks. bioRxiv.

Kerdaffrec E, Filiault DL, Korte A, Sasaki E, Nizhynska V, Seren U, Nordborg M. 2016. Multiple alleles at a single locus control seed dormancy in Swedish Arabidopsis. Elife 5.

King EG, Merkes CM, McNeil CL, Hoofer SR, Sen S, Broman KW, Long AD, Macdonald SJ. 2012. Genetic dissection of a model complex trait using the Drosophila Synthetic Population Resource. Genome Res 22: 1558-1566. 
Kofler R, Langmüller AM, Nouhaud P, Otte KA, Schlötterer C. 2016. Suitability of Different Mapping Algorithms for Genome-wide Polymorphism Scans with Pool-Seq Data. G3 doi:10.1534/g3.116.034488.

Kofler R, Pandey RV, Schlötterer C. 2011. PoPoolation2: identifying differentiation between populations using sequencing of pooled DNA samples (Pool-Seq). Bioinformatics 27: 3435-3436.

Kopp M, Matuszewski S. 2014. Rapid evolution of quantitative traits: theoretical perspectives. Evol Appl 7: 169-191.

Langley CH, Tobari YN, Kojima KI. 1974. Linkage disequilibrium in natural populations of Drosophila melanogaster. Genetics 78: 921-936.

Langmead B, Salzberg SL. 2012. Fast gapped-read alignment with Bowtie 2. Nature methods 9: 357-359.

Langmüller AM, Nolte V, Galagedara R, Poupardin R, Dolezal M, Lečić S, S. S. 2020. Fitness effects for Ace insecticide resistance mutations are determined by ambient temperature. submitted.

Lee KM, Coop G. 2017. Distinguishing Among Modes of Convergent Adaptation Using Population Genomic Data. Genetics 207: 1591-1619.

Li H, Durbin R. 2010. Fast and accurate long-read alignment with Burrows-Wheeler transform. Bioinformatics 26: 589-595.

Li H, Handsaker B, Wysoker A, Fennell T, Ruan J, Homer N, Marth G, Abecasis G, Durbin R, Genome Project Data Processing S. 2009. The Sequence Alignment/Map format and SAMtools. Bioinformatics 25: 2078-2079.

Li J, Li H, Jakobsson M, Li S, Sjodin P, Lascoux M. 2012. Joint analysis of demography and selection in population genetics: where do we stand and where could we go? Mol Ecol 21: $28-44$.

Li YR, Keating BJ. 2014. Trans-ethnic genome-wide association studies: advantages and challenges of mapping in diverse populations. Genome Med 6: 91.

Lohmueller KE. 2014. The impact of population demography and selection on the genetic architecture of complex traits. PLoS Genet 10: e1004379.

Mallard F, Nolte V, Tobler R, Kapun M, Schlötterer C. 2018. A simple genetic basis of adaptation to a novel thermal environment results in complex metabolic rewiring in Drosophila. Genome biology 19: 119.

Mancuso N, Rohland N, Rand KA, Tandon A, Allen A, Quinque D, Mallick S, Li H, Stram A, Sheng $\mathrm{X}$ et al. 2016. The contribution of rare variation to prostate cancer heritability. Nat Genet 48: 30-35.

McCarthy MI, Abecasis GR, Cardon LR, Goldstein DB, Little J, Ioannidis JP, Hirschhorn JN. 2008. Genome-wide association studies for complex traits: consensus, uncertainty and challenges. Nat Rev Genet 9: 356-369.

Michalak P, Kang L, Schou MF, Garner HR, Loeschcke V. 2019. Genomic signatures of experimental adaptive radiation in Drosophila. Molecular ecology 28: 600-614.

Morgan TJ, Mackay TF. 2006. Quantitative trait loci for thermotolerance phenotypes in Drosophila melanogaster. Heredity 96: 232-242.

Novocraft. 2014. NovoAlign. doi:http://www.novocraft.com/products/novoalign/. 
852

Nuzhdin SV, Turner TL. 2013. Promises and limitations of hitchhiking mapping. Current opinion in genetics \& development 23: 694-699.

Otte KA, Schlötterer C. 2017. Polymorphism-aware protein databases - a prerequisite for an unbiased proteomic analysis of natural populations. Molecular ecology resources doi:10.1111/1755-0998.12656.

Otte KA, Schlötterer C. 2019. A generalised approach to detect selected haplotype blocks in Evolve and Resequence experiments. bioRxiv.

Palmieri N, Nolte V, Chen J, Schlötterer C. 2015. Genome assembly and annotation of a Drosophila simulans strain from Madagascar. Molecular ecology resources 15: 372-381.

Pavlidis P, Alachiotis N. 2017. A survey of methods and tools to detect recent and strong positive selection. J Biol Res (Thessalon) 24: 7.

Petavy G, David JR, Gibert P, Moreteau B. 2001. Viability and rate of development at different temperatures in Drosophila: a comparison of constant and alternating thermal regimes. $J$ Therm Biol 26: 29-39.

Pritchard JK, Pickrell JK, Coop G. 2010. The genetics of human adaptation: hard sweeps, soft sweeps, and polygenic adaptation. Current biology : $C B$ 20: R208-215.

R Core Team. 2019. R: A Language and Environment for Statistical Computing. R Foundation for Statistical Computing, Vienna, Austria (http://www.r-project.org/index.html).

Rako L, Blacket MJ, McKechnie SW, Hoffmann AA. 2007. Candidate genes and thermal phenotypes: identifying ecologically important genetic variation for thermotolerance in the Australian Drosophila melanogaster cline. Molecular ecology 16: 2948-2957.

Reid NM, Proestou DA, Clark BW, Warren WC, Colbourne JK, Shaw JR, Karchner SI, Hahn ME, Nacci D, Oleksiak MF et al. 2016. The genomic landscape of rapid repeated evolutionary adaptation to toxic pollution in wild fish. Science 354: 1305-1308.

Roesti M, Gavrilets S, Hendry AP, Salzburger W, Berner D. 2014. The genomic signature of parallel adaptation from shared genetic variation. Mol Ecol 23: 3944-3956.

Sachdeva H, Barton NH. 2018. Introgression of a Block of Genome Under Infinitesimal Selection. Genetics 209: 1279-1303.

Schlötterer C, Tobler R, Kofler R, Nolte V. 2014. Sequencing pools of individuals - mining genome-wide polymorphism data without big funding. Nature reviews Genetics 15: 749763.

Seabra SG, Fragata I, Antunes MA, Faria GS, Santos MA, Sousa VC, Simoes P, Matos M. 2018. Different Genomic Changes Underlie Adaptive Evolution in Populations of Contrasting History. Mol Biol Evol 35: 549-563.

Sella G, Barton NH. 2019. Thinking About the Evolution of Complex Traits in the Era of Genome-Wide Association Studies. Annu Rev Genomics Hum Genet 20: 461-493.

Shahandeh MP, Turner TL. 2020. The complex genetic architecture of male mate choice evolution between Drosophila species. Heredity (Edinb) 124: 737-750.

Shi H, Kichaev G, Pasaniuc B. 2016. Contrasting the Genetic Architecture of 30 Complex Traits from Summary Association Data. Am J Hum Genet 99: 139-153.

Simons YB, Turchin MC, Pritchard JK, Sella G. 2014. The deleterious mutation load is insensitive to recent population history. Nat Genet 46: 220-224. 
Smit AFA, Hubley R, Green P. 2015. RepeatMasker Open-4.0 (2013-2015) (http://www.repeatmasker.org/).

Spitzer K, Pelizzola M, Futschik A. 2020. Modifying the Chi-square and the CMH test for population genetic inference: Adapting to overdispersion. Ann Appl Stat 14: 202-220.

Stajich JE, Hahn MW. 2005. Disentangling the effects of demography and selection in human history. Mol Biol Evol 22: 63-73.

Stam LF, Laurie CC. 1996. Molecular dissection of a major gene effect on a quantitative trait: the level of alcohol dehydrogenase expression in Drosophila melanogaster. Genetics 144: $1559-1564$.

Storz JF. 2005. Using genome scans of DNA polymorphism to infer adaptive population divergence. Molecular ecology 14: 671-688.

Tajima F. 1989. Statistical method for testing the neutral mutation hypothesis by DNA polymorphism. Genetics 123: 585-595.

Takata R, Takahashi A, Fujita M, Momozawa Y, Saunders EJ, Yamada H, Maejima K, Nakano K, Nishida Y, Hishida A et al. 2019. 12 new susceptibility loci for prostate cancer identified by genome-wide association study in Japanese population. Nat Commun 10: 4422.

Taus T, Futschik A, Schlötterer C. 2017. Quantifying Selection with Pool-Seq Time Series Data. Molecular biology and evolution 34: 3023-3034.

Thornton KR. 2019. Polygenic Adaptation to an Environmental Shift: Temporal Dynamics of Variation Under Gaussian Stabilizing Selection and Additive Effects on a Single Trait. Genetics doi:10.1534/genetics.119.302662.

Tobler R, Franssen SU, Kofler R, Orozco-Terwengel P, Nolte V, Hermisson J, Schlötterer C. 2014. Massive habitat-specific genomic response in D. melanogaster populations during experimental evolution in hot and cold environments. Molecular biology and evolution 31: 364-375.

Tobler R, Nolte V, Schlotterer C. 2017. High rate of translocation-based gene birth on the Drosophila Y chromosome. Proceedings of the National Academy of Sciences of the United States of America 114: 11721-11726.

Turner TL, Bourne EC, Von Wettberg EJ, Hu TT, Nuzhdin SV. 2010. Population resequencing reveals local adaptation of Arabidopsis lyrata to serpentine soils. Nature genetics 42: 260-263.

Visscher PM, Macgregor S, Benyamin B, Zhu G, Gordon S, Medland S, Hill WG, Hottenga JJ, Willemsen G, Boomsma DI et al. 2007. Genome partitioning of genetic variation for height from 11,214 sibling pairs. Am J Hum Genet 81: 1104-1110.

Vitti JJ, Grossman SR, Sabeti PC. 2013. Detecting natural selection in genomic data. Annual review of genetics 47: 97-120.

Vlachos C, Kofler R. 2018. MimicrEE2: Genome-wide forward simulations of Evolve and Resequencing studies. PLoS computational biology 14: e1006413.

Wojcik GL, Graff M, Nishimura KK, Tao R, Haessler J, Gignoux CR, Highland HM, Patel YM, Sorokin EP, Avery CL et al. 2019. Genetic analyses of diverse populations improves discovery for complex traits. Nature 570: 514-518. 
Wu Y, Waite LL, Jackson AU, Sheu WH, Buyske S, Absher D, Arnett DK, Boerwinkle E, Bonnycastle LL, Carty CL et al. 2013. Trans-ethnic fine-mapping of lipid loci identifies population-specific signals and allelic heterogeneity that increases the trait variance explained. PLoS Genet 9: e1003379.

Xu S. 2003. Theoretical basis of the Beavis effect. Genetics 165: 2259-2268.

941 Yang J, Manolio TA, Pasquale LR, Boerwinkle E, Caporaso N, Cunningham JM, de Andrade M, Feenstra B, Feingold E, Hayes MG et al. 2011. Genome partitioning of genetic variation for complex traits using common SNPs. Nat Genet 43: 519-525.

Yeaman S, Whitlock MC. 2011. The genetic architecture of adaptation under migration-selection balance. Evolution; international journal of organic evolution 65: 1897-1911.

Zan Y, Carlborg O. 2019. A Polygenic Genetic Architecture of Flowering Time in the Worldwide Arabidopsis thaliana Population. Mol Biol Evol 36: 141-154.

Zan Y, Sheng Z, Lillie M, Ronnegard L, Honaker CF, Siegel PB, Carlborg O. 2017. Artificial Selection Response due to Polygenic Adaptation from a Multilocus, Multiallelic Genetic Architecture. Molecular biology and evolution 34: 2678-2689.

Zeng J, de Vlaming R, Wu Y, Robinson MR, Lloyd-Jones LR, Yengo L, Yap CX, Xue A, Sidorenko J, McRae AF et al. 2018. Signatures of negative selection in the genetic architecture of human complex traits. Nature genetics 50: 746-753.

Zhang XS, Hill WG. 2005. Predictions of patterns of response to artificial selection in lines derived from natural populations. Genetics 169: 411-425. 